\title{
Capacity Planning of Electric Car Charging Station Based on Discrete Time Observations and MAP(2)/G/c Queue
}

\author{
Csaba Farkas ${ }^{1 *}$, Miklós Telek² \\ 1 Department of Electric Power Engineering, Faculty of Electrical Engineering and Informatics, \\ Budapest University of Technology and Economics, H-1111 Budapest, Egry József u. 18., Hungary \\ 2 Department of Networked Systems and Services, Faculty of Electrical Engineering and Informatics, \\ Budapest University of Technology and Economics, H-1117 Budapest, Magyar Tudósok körútja 2., Hungary \\ *Corresponding author, e-mail: farkas.csaba@vet.bme.hu
}

Received: 17 December 2017, Accepted: 08 May 2018, Published online: 01 June 2018

\begin{abstract}
The modeling of electric car charging stations is essential for determining the required number of chargers in order to ensure the required service quality. In this paper we propose a new estimation method for the stochastic modeling of electric car charging stations, based on Markov arrival process (MAP).

The input of the proposed model is empirical data for the arrival and service process of electric cars, given as histograms: the number of arriving cars during a fixed time slot ( 5 minutes in our case) and the histogram of service times (in 5 minutes granularity). The fact that observations on the continuous time process of car charging are available in discrete time steps poses a modeling challenge, which was not considered before. We propose a procedure to fit the observed data with a continuous time MAP of order 2 such that three moments and a correlation parameter of the discrete time observations are matched with three moments and the correlation parameter of the continuous time MAP for the given time interval. We implemented the fitting procedure in MATLAB and verified the obtained model of car charging station against simulation. As the MAP model of the arrival processes is reasonably close to the observations, the obtained MAP/G/c queue allows a more accurate dimensioning of car charging station than the previously applied ones.
\end{abstract}

Keywords

electric vehicle, charging station, Markov arrival process, point process fitting, simulation

\section{Introduction}

Electric vehicles (EVs) require an adequate number of chargers at charging stations in order to have neither unwanted long queues at the station, nor a poorly utilized system due to idleness. Furthermore, without enough chargers the customers will not buy EVs out of sheer fear of range anxiety (i.e. they will not find a charging station nearby where they can recharge their cars when needed), while if there are not enough EVs, there is no point in constructing charging stations. To cope with this problem, government or industrial subsidies are required, both in promoting the dissemination of EVs and in constructing charging stations. This paper intends to suggest a modeling procedure that would help in the charging station construction by determining the required number of chargers in a fast charging station. As this topic is highly relevant today, many papers deal with it. They can be classified into two sets: some papers use optimization algorithms to dimension a charging station: for example [1] proposes a multi-objective electric vehicle charging station planning method which can ensure charging service while reducing power losses and voltage deviations of distribution systems; authors of [2] study the EV charging station placement problem by finding the best locations to construct charging stations in a city in such a way, that they minimize the construction cost with coverage extended to the whole city and they also study the complexity of the station placement problem; authors of [3] developed a mathematical model for the optimal sizing of EV charging stations with the minimization of total cost associated to these stations and solved it by a modified primal-dual interior point algorithm.

Other papers use stochastic models, with which system performance can be studied: the literature review shows that they almost exclusively use $\mathrm{M} / \mathrm{M} / \mathrm{c}$ queues for charging station modeling, i.e., they assume the arrival and service process 
of EVs to be Poisson-processes. In this sense [4] models the fast charging stations with the help of an $\mathrm{M} / \mathrm{G} / \mathrm{S} / \mathrm{K}$ queue and incorporates a fast charging model (i.e. charging characteristics show that charging power during fast charging is not constant) into the queuing analysis as well as the revenue model of the charging station; the authors of [5] present a methodology of modeling the overall charging demand of plug-in hybrid electric vehicles (PHEVs) and employ queuing theory $(\mathrm{M} / \mathrm{M} / \mathrm{c}$ model) to describe the behavior of multiple PHEVs; [6] uses a discrete-state, discrete-time Markov chain to define the states of all the EVs at each time step. Four states are considered in [6], depending on whether an $\mathrm{EV}$ is parked or not and on the parking location.

However, as [7] clearly states, most of the M/M/c models are based on some unrealistic assumptions without validation. [4] rejects the assumption that the service process can be modeled as a Poisson-process, as charging time depends on initial battery state of charge. This statement is supported by measurements made by the authors of [8], see Fig. 1. Furthermore, according to [9], sojourn times are in general not exponentially distributed. In fact, the assumption that EV charging can be modeled by $\mathrm{M} / \mathrm{M} / \mathrm{c}$ queues has to our best knowledge never really been justified in the literature.

Our aim in this paper is thus to create a model that can be used even if the arrival and service processes are not Poisson-processes. We propose a stochastic model based on Markov arrival processes (MAP) to capture the nature of electric car charging process. The only paper we found using MAP is [10], where the authors use a DMAP/PH/N/R model (where DMAP stands for discrete time MAP and $\mathrm{PH}$ for phase type distributed service time) to investigate a battery replacement strategy to increase the efficiency of chargers and save drivers' time at charging stations.

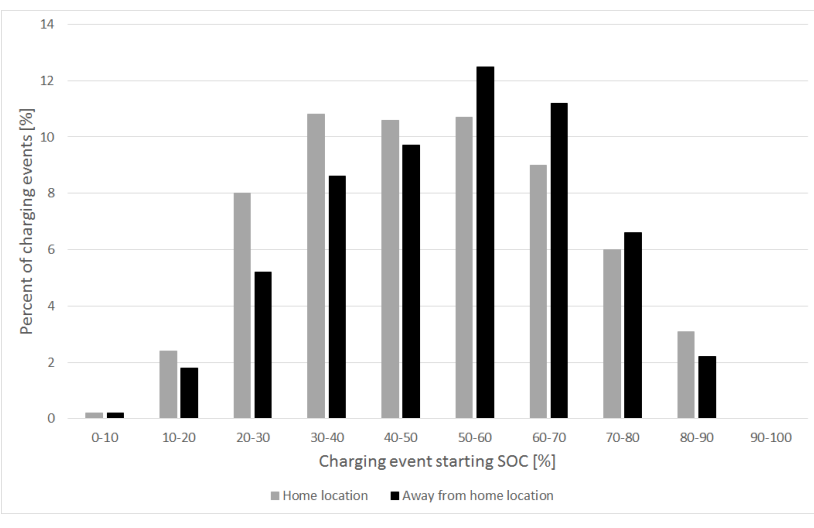

Fig. 1 Distribution of battery pack SOC at the start of charging by charging location [8]
However, our proposed approach is essentially different. We assume that cars arrive according to a continuous time stochastic process to which we have observations only in equidistance discrete time instances. This assumption is motivated by the way automatic traffic counting is conducted: due to the large number of data, traffic counting is basically done in discrete time intervals, mainly in hourly resolution, but for design purposes, smaller intervals can also be used (see e.g. [11], where 20s resolution was also used). As no data is available regarding arrival times of EVs we take the input data from [12], where the motion of a taxi fleet composed of electric cars is simulated in $5 \mathrm{~min}$ long time periods. That is why we assume that the EV arrivals are known in every $\Delta=5 \mathrm{~min}$ long time periods. The fact that we need to fit a continuous time MAP to a set of discrete observation instances raises a new research challenge. To the best of our knowledge this problem is considered here for the first time. We would like to emphasize, that the main contribution of our paper is the applied methodology, which has not been applied for EV modelling yet and contains the solution of a previously unavailable analysis step (MAP fitting based on restricted observations), the numerical example in Section 5 is presented to show the capabilities of the proposed approach.

We would also like to emphasize that the paper does not intend to extend the investigations to charger station location planning, nor is its aim to give a comprehensive model including EV drivers' decision model, charging stations' pricing model, etc.

Furthermore, the number of cars arriving during a certain time period is largely stochastic and is driven by various factors, such as time of day, day of week, weather, etc. We did not explicitly taken consideration about these factors, but they are included implicitly in the model: the empirical data regarding car arrivals carry information about them, as for example the number of cars arriving at once is higher during peak hours and lower during other times of the day: as the latter occurs more often during the day, it is represented by higher probabilities, hence larger values in the histogram (see Fig. 3).

The rest of the paper is structured as follows. Section 2 summarizes the basics of MAP modeling. In Section 3 we present the theoretical basis of restricted observation based MAP fitting and in Section 4 the procedure itself. In Section 5 simulation results are presented and discussed. The paper is concluded in Section 6 . 


\section{Modeling with Markov arrival process}

This section introduces the basic properties of MAPs.

\subsection{Markov arrival processes}

The Markov arrival process is a point process where the arrivals are governed by a background continuous time Markov chain (CTMC). A possible interpretation of MAP is through the joint stochastic process $\{(N(t), J(t)): t \geq 0\}$ where $N(t)$ denotes the number of arrivals in the time interval $(0, t)$ and $J(t)$ denotes the state of the background CTMC (commonly referred to as phase) at time $t . J(t)$ is CTMC on the finite state space $M$, while $N(t)$ is a stochastic counting process depending on $J(t)$. The $(N(\cdot), J(\cdot))$ joint stochastic process is a CTMC on the state space $\{(n, j): n \geq$ $0,1 \leq j \leq M\}$ with the infinitesimal generator matrix

$$
Q=\left[\begin{array}{cccc}
D_{0} & D_{1} & 0 & 0 \\
0 & D_{0} & D_{1} & 0 \\
0 & 0 & D_{0} & D_{1} \\
\vdots & \vdots & 0 & \ddots
\end{array}\right],
$$

where

- $D_{0}$ and $D_{1}$ are $M \times M$ matrices,

- $D_{1} \geq 0$ elementwise,

- $\left[D_{0}\right]_{i, j} \geq 0,1 \leq i \neq j \leq M$ and $\left[D_{0}\right]_{i, i}<0,1 \leq i \leq M$,

- and $\sum_{j}\left[D_{0}\right]_{i, j}+\sum_{j}\left[D_{1}\right]_{i, j}=0$ that is

$\left(D_{0}+D_{1}\right) \cdot \mathbf{1}=0$

where $\mathbf{1}$ is the column vector of ones of the appropriate size.

This means that a Markov arrival process is defined by the matrices $D_{0}$ and $D_{1}$, where the elements of $D_{0}$ represent hidden transitions and elements of $D_{1}$ observable transitions.

We use second-order MAPs (denoted with MAP(2)) because they have significantly more modeling flexibility (e.g. correlated inter-arrival time) compared to Poisson processes while their computational complexity is still low. An important advantage of using MAP(2) is the availability of a canonical representation [13], which is a minimal unique Markovian representation for all members of the MAP(2) class. This means that $M=2$, and both $D_{0}$ and $D_{1}$ are $2 \times 2$ matrices.

Depending on whether the correlation of consecutive inter-arrivals is positive or negative there are two different canonical forms [13]. Based on the properties of our data sets (which are discussed in Subsection 2.3) we use only the canonical form with positive correlation in this paper.
The $D_{0}$ and $D_{1}$ matrix representation of this canonical form is as follows:

$D_{0}=\left[\begin{array}{cc}-\lambda_{1} & (1-a) \cdot \lambda_{1} \\ 0 & -\lambda_{2}\end{array}\right]$,

$D_{1}=\left[\begin{array}{cc}a \cdot \lambda_{1} & 0 \\ (1-b) \cdot \lambda_{2} & b \cdot \lambda_{2}\end{array}\right]$,

where $\lambda_{1}$ and $\lambda_{2}$ are rate parameters, $a$ and $b$ are probabilities. The transition graph representation of this canonical form is depicted on Fig. 2.

The stationary distribution of the MAP arrivals in a $\Delta$ long time interval is given by the following z-transform expression:

$p(\Delta, z)=\alpha \cdot e^{\left(D_{0}+D_{1} \cdot z\right) \cdot \Delta} \cdot \mathbf{1}$,

where $\alpha=\left[\left(\begin{array}{ll}\alpha_{0} & \alpha_{1}\end{array}\right)\right]$ is the time stationary phase distribution vector, and $\mathbf{1}=\left[\begin{array}{l}1 \\ 1\end{array}\right]$ is the summation vector of size 2. $\alpha$ is obtained from the $D_{0}$ and $D_{1}$ matrix representation as the solution of the linear system of equations

$\alpha \cdot\left(D_{0}+D_{1}\right)=0, \quad \alpha \cdot \mathbf{1}=1$,

and it is

$\alpha=\left[\begin{array}{cc}\frac{(1-b) \cdot \lambda_{2}}{(1-a) \cdot \lambda_{1}+(1-b) \cdot \lambda_{2}} & \frac{(1-a) \cdot \lambda_{1}}{(1-a) \cdot \lambda_{1}+(1-b) \cdot \lambda_{2}}\end{array}\right]$.

\subsection{Histogram of the empirical arrival process}

The number of cars arriving to the charging station during a fixed time slot is depicted on Fig. 3 (the example obtained from [12]).

We form the following z-transform polynomial from the histogram:

$$
A(z)=\sum_{i} p_{i} \cdot z^{i}
$$

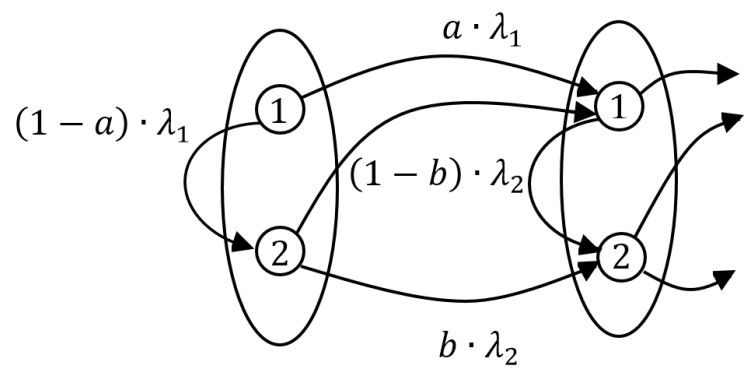

Fig. 2 Markov chain of the canonical MAP(2) with positive correlation 


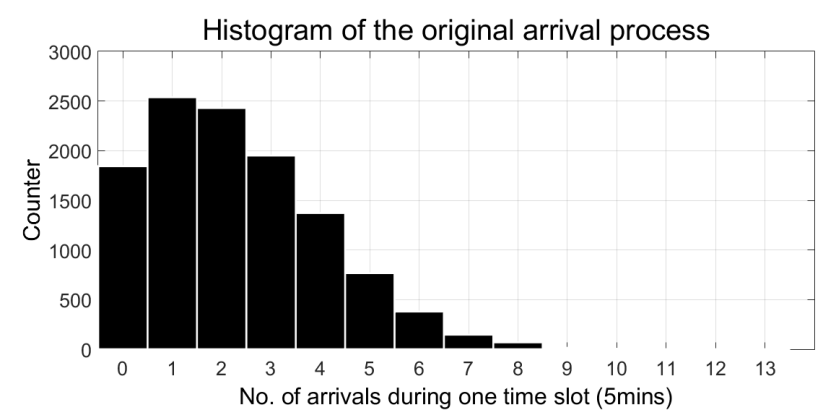

Fig. 3 Histogram showing the number of cars arriving in a $\Delta$ long time interval (example)

where $p_{i}$ is the probability that $i$ cars arrive in a $\Delta$ long time interval. The polynomial in our example (according to Fig. 3.) is the following:

$$
A(z)=\frac{1}{11521} \cdot\left(\begin{array}{c}
z^{13}+z^{12}+3 z^{11}+7 z^{10}+21 z^{9}+63 z^{8} \\
+149 z^{7}+383 z^{6}+765 z^{5}+1326 z^{4} \\
+2023 z^{3}+2393 z^{2}+2525 z+1861
\end{array}\right) \text {. }
$$

We fit the number of arrivals of a $\operatorname{MAP}(2)$ in a $\Delta$ long time interval, given in the form of Eq. (4), to this polynomial. More precisely we set the first three factorial moments of this data set, which can be obtained from $A(z)$ (as well as from the probabilities $p_{i}$, but we use $A(z)$ in order to exploit the similarity with the transform domain based computation of MAP(2) parameters) through its derivatives with respect to $z$, which are

$$
\begin{aligned}
&\left.\frac{d}{d z} A(z)\right|_{z=1}=2.315, \\
& \text { - }\left.\frac{d^{2}}{d z^{2}} A(z)\right|_{z=1}=6.2644, \\
& \text { - }\left.\frac{d^{3}}{d z^{3}} A(z)\right|_{z=1}=18.2198 .
\end{aligned}
$$

\subsection{Correlation of the arrival data}

The number of car arrivals in consecutive $\Delta$ long time intervals can be independent or dependent. We check the dependence structure of the car arrival process by computing the experimental correlation of observation intervals $\mathrm{k}$ lags apart. Having $N$ samples the lag-k correlation is computed between the first $N-k$ observations: $x_{1}, x_{2}, \ldots, x_{N-k}$ and the next $N-k$ observations $x_{k+1}, x_{k+2}, \ldots, x_{N}$ according to the following expression [15]

$$
\hat{\rho}_{k}=\frac{\sum_{t=1}^{N-k}\left(x_{t}-\bar{x}_{(1)}\right) \cdot\left(x_{t+1}-\bar{x}_{(k+1)}\right)}{\sqrt{\sum_{t=1}^{N-k}\left(x_{t}-\bar{x}_{(1)}\right)^{2} \cdot \sum_{t=k+1}^{N}\left(x_{t}-\bar{x}_{(k+1)}\right)^{2}}},
$$

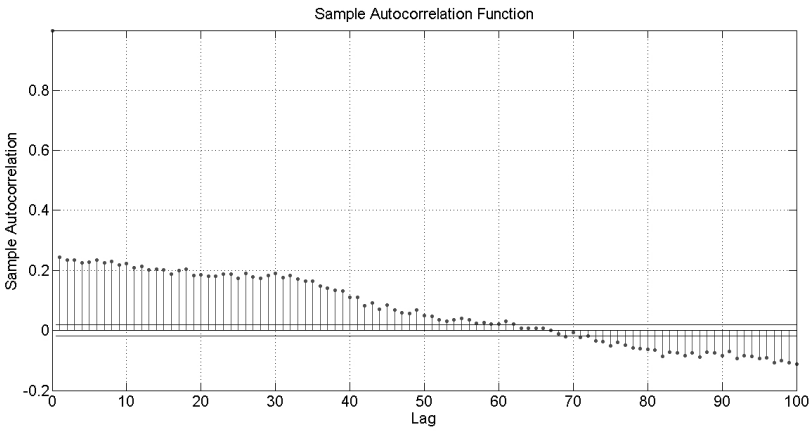

Fig. 4 Correlation of the original dataset

where $\bar{x}_{(1)}$ is the experimental mean of the first $N-k$ observations and $\bar{x}_{(k+1)}$ is the experimental mean of the last $N-k$ observations.

The correlation of the data sample for arrivals can also be obtained by using MATLAB's autocorr function. The experimental lag-k correlation parameters are depicted in Fig. 4. The lag-1 correlation is $\hat{\rho}_{1}=0.2443$.

To emphasize the applicability of the proposed method we note that the $\operatorname{MAP}(2)$ class can also represent the case when the correlation is zero. In that case parameter $b$ equals to zero, thus the canonical form of MAP(2) simplifies to

$D_{1}=\left[\begin{array}{cc}a \cdot \lambda_{1} & 0 \\ \lambda_{2} & 0\end{array}\right]$,

while $D_{0}$ remains the same.

\subsection{Moment matching procedure}

The MAP(2) canonical form has four unknown parameters $\left(a, b, \lambda_{1}, \lambda_{2}\right)$. We set these parameters such that the first three moments of the inter-arrival time distribution $\left(\hat{m}_{k}, k=1,2,3\right)$, and the lag- 1 correlation of the experimental data $\left(\hat{\rho}_{1}\right)$ is matched. This procedure requires calculating these four parameters from both the empirical data and the $\operatorname{MAP}(2)$ canonical from. For the latter ones we need the first 3 derivatives of $p(\Delta, z)$ with respect to $z$ at $z=1$ and the lag-1 correlation from the double transform description of the number of car arrivals in consecutive intervals given in Eq. (23). Finally, we have to solve the obtained system of equation for the variables $a, b, \lambda_{1}$ and $\lambda_{2}$.

\section{Derivatives of $p(\Delta, z)$ and the correlation}

Although theoretically the symbolic derivation of the $p(\Delta, z)$ polynomial is possible, but it is computationally challenging. Instead of the direct, brute force solution, we apply some algebraic manipulations to make computations faster (and feasible). 


\subsection{First moment}

Only the part $e^{\left(D_{0}+D_{1} \cdot z\right) \cdot \Delta}$ contains the parameter $z$ in Eq. (4), thus we have to calculate $\left.\frac{d}{d z} e^{\left(D_{0}+D_{1} \cdot z\right) \cdot \Delta}\right|_{z=1}$. The Taylor-series expansion of the matrix exponential function is

$$
\begin{aligned}
& \frac{d}{d z} e^{\left(D_{0}+D_{1} \cdot z\right) \cdot \Delta}=\frac{d}{d z} \sum_{i=0}^{\infty} \frac{\Delta^{i}}{i !} \cdot\left(D_{0}+D_{1} \cdot z\right)^{i} \\
& =\sum_{i=0}^{\infty} \frac{\Delta^{i}}{i !} \cdot \frac{d}{d z}\left(D_{0}+D_{1} \cdot z\right)^{i} .
\end{aligned}
$$

Due to the matrices in the series, the order of the parts matter this time. The calculation yields

$$
\begin{aligned}
& \left.\frac{d}{d z} e^{\left(D_{0}+D_{1} \cdot z\right) \cdot \Delta}\right|_{z=1}=\sum_{i=1}^{\infty} \frac{\Delta^{i}}{i !} \cdot \sum_{k=0}^{i-1}\left(D_{0}+D_{1} \cdot z\right)^{k} D_{1} \\
& \left.\cdot\left(D_{0}+D_{1} \cdot z\right)^{i-k-1}\right|_{z=1},
\end{aligned}
$$

so

$$
\begin{aligned}
& p(\Delta, z)=\alpha \cdot e^{\left(D_{0}+D_{1} \cdot z\right) \cdot \Delta} \cdot \mathbf{1} \\
& =\left.\alpha \cdot \sum_{i=1}^{\infty} \frac{\Delta^{i}}{i !} \cdot \sum_{k=0}^{i-1}\left(D_{0}+D_{1} \cdot z\right)^{k} D_{1} \cdot\left(D_{0}+D_{1} \cdot z\right)^{i-k-1}\right|_{z=1} \cdot \mathbf{1} \\
& =\alpha \cdot \sum_{i=1}^{\infty} \frac{\Delta^{i}}{i !} \cdot \sum_{k=0}^{i-1}\left(D_{0}+D_{1}\right)^{k} D_{1} \cdot\left(D_{0}+D_{1}\right)^{i-k-1} \cdot \mathbf{1} .
\end{aligned}
$$

This means that we can simplify Eq. (12) further as follows:

$$
\left.\frac{d}{d z} p(\Delta, z)\right|_{z=1}=\alpha \cdot \Delta \cdot D_{1} \cdot \mathbf{1} \text {. }
$$

This formula gives the first moment.

\subsection{Second moment}

The calculation is similar to the first moment: we want to obtain $\left.\frac{d^{2}}{d z^{2}} e^{\left(D_{0}+D_{1} \cdot z\right) \cdot \Delta}\right|_{z=1}$, so we take the derivation of Eq. (11) with respect to $z$, and we obtain

$$
\begin{aligned}
& \frac{d^{2}}{d z^{2}} e^{\left(D_{0}+D_{1} \cdot z\right) \cdot \Delta} \\
& =\frac{d}{d z}\left[\sum_{i=1}^{\infty} \frac{\Delta^{i}}{i !} \cdot\left(D_{0}+D_{1} \cdot z\right)^{k} \cdot D_{1} \cdot\left(D_{0}+D_{1} \cdot z\right)^{i-k-1}\right] .
\end{aligned}
$$

After the Taylor-series expansion and the derivation of the first few parts we can see that the solution is

$$
\begin{aligned}
& \frac{d^{2}}{d z^{2}} e^{\left(D_{0}+D_{1} \cdot z\right) \cdot \Delta}=\sum_{i=2}^{\infty} \frac{\Delta^{i}}{i !} \\
& \left(\begin{array}{l}
\sum_{k=1}^{i-1} \sum_{l=0}^{k-1}\left(D_{0}+D_{1} \cdot z\right)^{l} \cdot D_{1} \cdot\left(D_{0}+D_{1} \cdot z\right)^{k-l-1} \\
\cdot D_{1} \cdot\left(D_{0}+D_{1} \cdot z\right)^{i-k-1}+\sum_{k=0}^{i-2 i-k-2} \sum_{l=0}\left(D_{0}+D_{1} \cdot z\right)^{k} \\
\cdot D_{1} \cdot\left(D_{0}+D_{1} \cdot z\right)^{l} \cdot D_{1} \cdot\left(D_{0}+D_{1} \cdot z\right)^{i-k-l-2}
\end{array}\right) .
\end{aligned}
$$

After further simplifications using Eq. (1) and Eq. (5) we obtain

$$
\begin{aligned}
& \left.\alpha \cdot \frac{d^{2}}{d z^{2}} e^{\left(D_{0}+D_{1} \cdot z\right) \cdot \Delta}\right|_{z=1} \cdot \mathbf{1} \\
& =2 ! \cdot \alpha \cdot \sum_{i=2}^{\infty} \frac{\Delta^{i}}{i !} \cdot D_{1} \cdot\left(D_{0}+D_{1}\right)^{i-2} \cdot D_{1} \cdot \mathbf{1} .
\end{aligned}
$$

Let's denote $D_{0}+D_{1}$ with $D$. This means that we can reformulate Eq. (16) as

$$
2 ! \cdot \alpha \cdot D_{1} \cdot \sum_{i=2}^{\infty} \frac{\Delta^{i}}{i !} \cdot D^{i-2} \cdot D_{1} \cdot \mathbf{1},
$$

where we can see that $\sum_{i=2}^{\infty} \frac{\Delta^{i}}{i !} \cdot D^{i-2}$ resembles to the Taylorseries expansion of the matrix exponential function $e^{D \Delta}$. To obtain that formula, we have to alter Eq. (17) a little, but we cannot extend the formula by multiplying simply with $D^{2} \cdot \mathrm{D}^{-2}$, because $\mathrm{D}^{-2}$ does not exist as $D$ is singular (see Eq. (1)). Instead, we have to do the extension using $(D-1 \cdot \alpha)^{2} \cdot(D-1 \cdot \alpha)^{-2}$. If $D$ is an irreducible Markovchain, then $\mathrm{D}-\mathbf{1} \cdot \alpha$ is not singular [15]. With further calculations, utilizing Eq. (1) and Eq. (5), we can reformulate Eq. (17) as

$\left.\alpha \cdot \frac{d^{2}}{d z^{2}} e^{\left(D_{0}+D_{1} \cdot z\right) \cdot \Delta}\right|_{z=1} \cdot \mathbf{1}=2$ !

$\left[\begin{array}{l}\alpha \cdot D_{1} \cdot \frac{\Delta^{2}}{2 !} \cdot D_{1} \cdot \mathbf{1}+\alpha \cdot D_{1} \\ \cdot\left(e^{D \cdot \Delta}-I-D \cdot \Delta-\frac{(D \cdot \Delta)^{2}}{2 !}\right) \cdot(D-\mathbf{1} \cdot \alpha)^{-2} \cdot D_{1} \cdot \mathbf{1}\end{array}\right]$.

This is the second momentum of the number of arrivals in $(0, \Delta)$, where $I$ denotes the $2 \times 2$ identity matrix.

\subsection{Third moment}

Based on our calculations regarding the first and the second moment, we can determine the third one. We have seen that there was a single summation in the case of the first moment, a double summation in the second and here in the case of the third moment, a triple summation would come, with the argument being something like $\left(D_{0}+D_{1} \cdot z\right)^{k}$ $\cdot D_{1} \cdot\left(D_{0}+D_{1} \cdot z\right)^{l} \cdot D_{1} \cdot\left(D_{0}+D_{1} \cdot z\right)^{m} \cdot D_{1} \cdot\left(D_{0}+D_{1} \cdot z\right)^{n}$.

This one, however is hard to deal with, as not all of the factors disappear when we multiply with the vectors $\alpha$ and $\mathbf{1}$, so convolutions would appear. To make calculations easier, we can trace the summation back to matrix products: if we raise to powers the

$\mathbb{D}_{1}=\left[\begin{array}{cc}D & D_{1} \\ 0 & D\end{array}\right]$ 
hyper matrix, we can obtain the factors in the aforementioned sums; they are given by the upper right block of the $\mathbb{D}_{1}$ hyper matrix, so we have to multiply $\mathbb{D}_{1}$ with $\left[\begin{array}{ll}I_{2} & 0_{2}\end{array}\right]$ from the left and with $\left[\begin{array}{l}0_{2} \\ I_{2}\end{array}\right]$ from the right, where $I_{2}$ is the $2 \times 2$ identity matrix and $0_{2}$ is the $2 \times 2$ zero matrix. Using the hyper matrix we can obtain the third momentum as follows:

$$
\begin{aligned}
& \left.\alpha \cdot \frac{d^{3}}{d z^{3}} e^{\left(D_{0}+D_{1} \cdot z\right) \cdot \Delta}\right|_{z=1} \cdot \mathbf{1}=3 ! \cdot \alpha \cdot D_{1} \cdot\left[\begin{array}{ll}
I_{2} & 0_{2}
\end{array}\right] \\
& \cdot \sum_{n=3}^{\infty} \frac{\Delta^{n}}{n !} \cdot \mathbb{D}_{1}^{n-2} \cdot \mathbb{D}_{e}^{2} \cdot \mathbb{D}_{e}^{-2} \cdot\left[\begin{array}{l}
0_{2} \\
I_{2}
\end{array}\right] \cdot D_{1} \cdot \mathbf{1},
\end{aligned}
$$

where

$$
\mathbb{D}_{e}=\left[\begin{array}{cc}
D-\mathbf{1} \cdot \alpha & D_{1} \\
0 & D-\mathbf{1} \cdot \alpha
\end{array}\right] .
$$

If we calculate the powers of the $\mathbb{D}_{e}$ hyper matrix and substitute the obtained results into Eq. (20), we can see that the third moment is simplified:

$$
\begin{aligned}
& \left.\alpha \cdot \frac{d^{3}}{d z^{3}} e^{\left(D_{0}+D_{1} \cdot z\right) \cdot \Delta}\right|_{z=1} \cdot \mathbf{1}=3 ! \cdot \alpha \cdot D_{1} \cdot\left[\begin{array}{ll}
I_{2} & 0
\end{array}\right] \cdot \sum_{n=3}^{\infty} \frac{\Delta^{n}}{n !} \\
& \cdot\left(\mathbb{D}_{1}^{n}+\left[\begin{array}{ll}
0 & D^{n-3} \cdot\left(-D \cdot D_{1} \cdot \mathbf{1} \cdot \alpha+D_{1} \cdot \mathbf{1} \cdot \alpha\right) \\
0 & 0
\end{array}\right]\right) \cdot \mathbb{D}_{e}^{-2} \\
& \cdot\left[\begin{array}{l}
0_{2} \\
I_{2}
\end{array}\right] \cdot D_{1} \cdot \mathbf{1} .
\end{aligned}
$$

The inner hyper matrix can be rewritten into matrix exponential form utilizing the summation, so we can obtain the formula for the third moment:

$$
\begin{aligned}
& \alpha \cdot D_{1} \cdot\left[\begin{array}{ll}
I_{2} & 0_{2}
\end{array}\right] \cdot \sum_{n=3}^{\infty} \frac{\Delta^{n}}{n !} \cdot \mathbb{D}_{1}^{n} \cdot \mathbb{D}_{e}^{-2} \cdot\left[\begin{array}{l}
0_{2} \\
I_{2}
\end{array}\right] \cdot D_{1} \cdot \mathbf{1} \\
& =\alpha \cdot D_{1} \cdot\left[\begin{array}{ll}
I_{2} & 0_{2}
\end{array}\right] \cdot\left(e^{\mathbb{D}_{1} \cdot \Delta}-I_{4}-\mathbb{D}_{1} \cdot \Delta-\frac{\left(\mathbb{D}_{1} \cdot \Delta\right)^{2}}{2 !}\right) \\
& \cdot \mathbb{D}_{e}^{-2} \cdot\left[\begin{array}{l}
0_{2} \\
I_{2}
\end{array}\right] \cdot D_{1} \cdot \mathbf{1}
\end{aligned}
$$

is the matrix exponential form of Eq. (22) without the inner hyper matrix and

$$
\begin{aligned}
& -\left(e^{D \Delta}-I-D \Delta-\frac{(D \Delta)^{2}}{2 !}\right) \cdot(D-\mathbf{1} \cdot \alpha)^{-2} \cdot D_{1} \cdot \mathbf{1} \cdot \alpha, \\
& \cdot \frac{\Delta^{3}}{3 !} \cdot D_{1} \cdot \mathbf{1} \cdot \alpha+\left(e^{D \Delta}-I-D \Delta-\frac{(D \Delta)^{2}}{2 !}-\frac{(D \Delta)^{3}}{3 !}\right) \\
& \cdot(D-\mathbf{1} \cdot \alpha)^{-3} \cdot D_{1} \cdot \mathbf{1} \cdot \alpha
\end{aligned}
$$

are the additional terms obtained from the inner hyper matrix. With this, the third moment is also given.

\subsection{Correlation}

The correlation is calculated from the joint probability distribution of the number of cars arrived in the first and the second 5 minute time step. Let $P\left(z_{1} z_{2}\right)$ denote the z-transform of the joint probability, then

$P\left(z_{1} z_{2}\right)=\alpha \cdot e^{\left(D_{0}+D_{1} \cdot z_{1}\right) \cdot \Delta} \cdot e^{\left(D_{0}+D_{1} \cdot z_{2}\right) \cdot \Delta} \cdot \mathbf{1}$.

From this probability we can calculate the expected value of these variables as follows:

$E\left(x_{1} x_{2}\right)=\left.\frac{\partial}{\partial z_{1}} \frac{\partial}{\partial z_{2}} P\left(z_{1} z_{2}\right)\right|_{z_{1}=z_{2}=1}$.

The correlation is obtained from the expected value as follows:

$\operatorname{corr}=\frac{E\left(x_{1} x_{2}\right)-E\left(x_{1}\right) \cdot E\left(x_{2}\right)}{\sigma_{x_{1}} \cdot \sigma_{x_{2}}}$

where $\sigma_{X_{1}}$ and $\sigma_{X_{2}}$ are the variances of the random values $x_{1}$ and $x_{2}$. As $x_{1}$ and $x_{2}$ represent the number of arriving cars in the first and the second time slot, respectively, and the investigated process is assumed to be stationary, the variances are equal to each other and can be calculated from the moments as shown in Eq. (26):

$$
\sigma^{2}=E\left(x_{1}^{2}\right)-\left(E\left(x_{1}\right)\right)^{2} \text {. }
$$

We have already calculated all the required parameters before (see Eqs. (13), (18) and (22)), so the correlation is

$$
\operatorname{corr}=\frac{\left.\alpha \cdot D_{1} \cdot\left\{\begin{array}{l}
{\left[e^{D \cdot \Delta}-I-\Delta \cdot \mathbf{1} \cdot \alpha\right)} \\
\cdot(D-\mathbf{1} \cdot \alpha)^{-1}
\end{array}\right]^{2}-\Delta^{2} \cdot \mathbf{1} \cdot \alpha\right\} \cdot D_{1} \cdot \mathbf{1}}{\alpha \cdot D_{1} \cdot\left[\begin{array}{l}
\Delta^{2} \cdot(I-\mathbf{1} \cdot \alpha)+2 ! \\
\left.\cdot\left(e^{D \cdot \Delta}-I-D \cdot \Delta-\frac{(D \cdot \Delta)^{2}}{2 !}\right)\right] \cdot D_{1} \cdot \mathbf{1} \\
\cdot(D-\mathbf{1} \cdot \alpha)^{-2}
\end{array}\right]} .
$$

\section{The matching procedure}

We have obtained the symbolic forms of the first three moments of the $p(\Delta, z)$ polynomial and the corr parameter. Now, we have to solve the system of non-linear equations 


$$
\left\{\begin{array}{l}
\left.\frac{d}{d z} p(\Delta, z)\right|_{z=1}=\left.\frac{d}{d z} A(z)\right|_{z=1}=2.315 \\
\left.\frac{d^{2}}{d z^{2}} p(\Delta, z)\right|_{z=1}=\left.\frac{d^{2}}{d z^{2}} A(z)\right|_{z=1}=6.2644 \\
\left.\frac{d^{3}}{d z^{3}} p(\Delta, z)\right|_{z=1}=\left.\frac{d^{3}}{d z^{3}} A(z)\right|_{z=1}=18.2198 \\
\text { corr }=\hat{\rho}_{1}=0.2443
\end{array}\right.
$$

for the variables $a, b, \lambda_{1}, \lambda_{2}$. Fortunately, the $f_{\text {solve func- }}$ tion of MATLAB managed to obtain the results thanks to the algebraic manipulations summarized in the previous section. Without those manipulations all of our attempts failed. For our data set the obtained solution was:

- $a=0.2971$,

- $b=0.6762$,

- $\lambda_{1}=0.3196$,

- $\lambda_{2}=0.9861$,

which gives a proper MAP(2) canonical form [13] with valid probability and rate values.

\subsection{The service process}

The service process can as well be modeled by a MAP(2) process: the fitting is done similarly, like before. In our example, however we constructed a simpler model for the service process as the histogram of the service time is much simpler, as depicted on Fig. 5.

It is clear from Fig. 5 that in this case the MAP(2) modeling would be preposterous: all we have to do is to determine the probabilities of each option (i.e. charging lasts for 5 or 6 time intervals) and raffle one of these numbers randomly, using the obtained probabilities for weighting. This is why the service process is considered to be $\mathrm{G}$ (general) instead of MAP(2) in our example. We have to note that this service process obtained from [12] implicitly incorporates the initial battery state of charge (SOC) of cars. For further applications data regarding battery SOC is also needed to be able to model the service process properly.

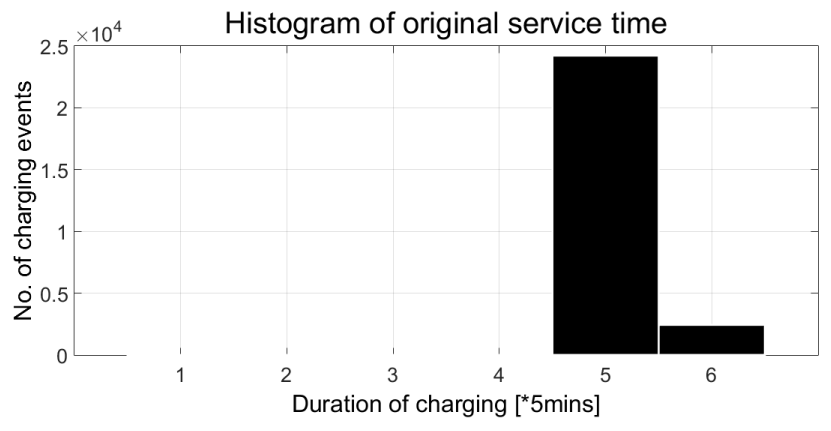

Fig. 5 Histogram of service time duration obtained from [12]

\section{Simulation of the electric car charging station}

We simulated the whole process using MATLAB. Cars arrive to charge according to the MAP(2) process with the calculated parameters.

If there is any available charger, they connect to it and begin charging and the charger becomes occupied. Charging time is raffled according to the service process as presented in Section 4.1. In every time step, the charging time left for a given car decreases and if it reaches 0 , the car is recharged, leaves the station and the charger becomes available again. If there is no available charger, the incoming cars have to wait, hence a waiting queue forms. The waiting queue has an FCFS discipline. For a given number of chargers we can determine the number of cars that have to wait (see Fig. 6 as an example). The aim is to have enough chargers in the charging station so that the probability of waiting is below a pre-defined threshold.

Running the simulation for 100 times we can determine the number of waiting cars for a given number of chargers (see Fig. 7).

To indicate the variance of the simulation we used MATLAB's boxplot function, where on each box, the central mark is the median, the edges of the box are the 25 th and 75 th percentiles, the whiskers extend to the most extreme data points not considered outliers, and outliers are plotted individually.

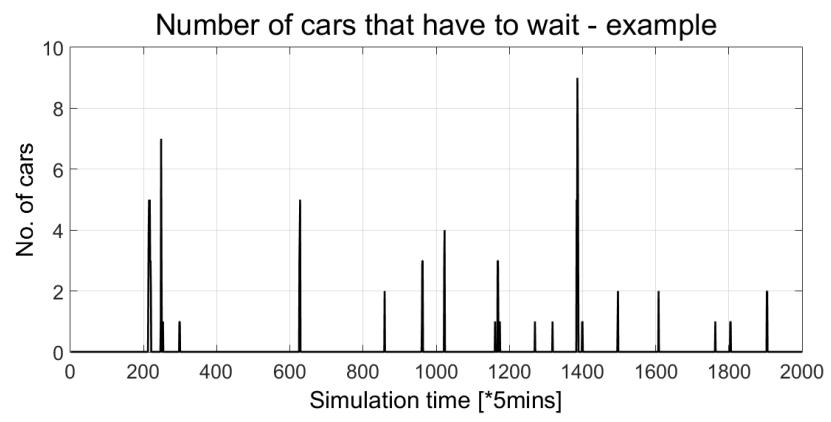

Fig. 6 Number of cars that have to wait - example

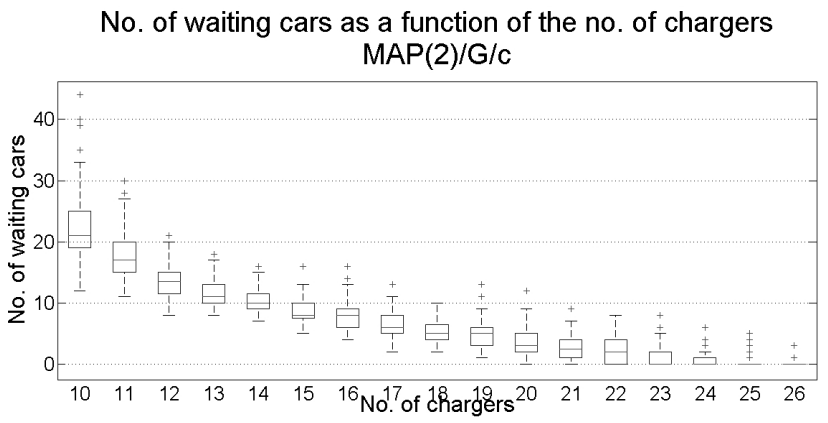

Fig. 7 Number of cars that have to wait for given no. of chargers 


\section{Conclusion}

The problem of appropriately dimensioned recharging units for electric vehicles is as important as appropriately dimensioned fueling units for cars with internal combustion engines. In this paper, exceeding the modeling restrictions of previously applied Poisson process based analyses, we addressed this dimensioning problem using

\section{References}

[1] Wang, G., Xu, Z., Wen, F., Wong, K. P. "Traffic-Constrained Multiobjective Planning of Electric-Vehicle Charging Stations", IEEE Transactions on Power Delivery, 28(4), pp. 2363-2372, 2013. https://doi.org/10.1109/TPWRD.2013.2269142

[2] Lam, A. Y. S., Leung, Y.-W., Chu, X. "Electric Vehicle Charging Station Placement: Formulation, Complexity, and Solutions", IEEE Transactions on Smart Grid, 5(6), pp. 2846-2856, 2014. https://doi.org/10.1109/TSG.2014.2344684

[3] Liu, Z., Wen, F., Ledwich, G. "Optimal Planning of Electric-Vehicle Charging Stations in Distribution Systems", IEEE Transactions on Power Delivery, 28(1), pp. 102-110, 2013. https://doi.org/10.1109/TPWRD.2012.2223489

[4] Fan, P., Sainbayar, B., Ren, S. "Operation Analysis of Fast Charging Stations With Energy Demand Control of Electric Vehicles", IEEE Transactions on Smart Grid, 6(4), pp. 1819-1826, 2015. https://doi.org/10.1109/TSG.2015.2397439

[5] Li, G., Zhang, X.-P. "Modeling of Plug-in Hybrid Electric Vehicle Charging Demand in Probabilistic Power Flow Calculations", IEEE Transactions on Smart Grid, 3(1), pp. 492-499, 2012. https://doi.org/10.1109/TSG.2011.2172643

[6] Soares, F. J., Peças Lopes, J. A., Rocha Almeida, P. M., Moreira, C. L., Seca, L. "A Stochastic Model to Simulate Electric Vehicles Motion and Quantify the Energy Required from the Grid", presented at 17th Power Systems Computation Conference, Stockholm, Sweden, Aug., 22-26., 2011.

[7] Zhang, X., Grijalva, S. "An Advanced Data Driven Model for Residential Electric Vehicle Charging Demand", 2015 IEEE Power \& Energy Society General Meeting, Denver, USA, Jul., 26-30., 2015. pp. 1-5. https://doi.org/10.1109/PESGM.2015.7286396

[8] Smart, J., Schey, S. "Battery Electric Vehicle Driving and Charging Behavior Observed Early in The EV Project", SAE International Journal of Alternative Powertrains, 1(1), pp. 27-33, 2012. https://doi.org/10.4271/2012-01-0199 continuous time MAPs assuming that only aggregate experimental data is available for time intervals of the same length. This limitation on the available data arises new modeling challenges for parameter matching of MAPs. We proposed a solution method using the canonical representation of MAP(2) processes.

[9] Rolink, J., Rehtanz, C. "Estimation of the availability of grid-connected electric vehicles by non-homogeneous semi-Markov processes", In: 2011 IEEE Trondheim PowerTech, Trondheim, Norway, Jun., 19-23., 2011. https://doi.org/10.1109/PTC.2011.6019403

[10] Dong, Q., Niyato, D., Wang, P., Han, Z. "The PHEV Charging Scheduling and Power Supply Optimization for Charging Stations", IEEE Transactions on Vehicular Technology, 65(2), pp. 566-580, 2016.

https://doi.org/10.1109/TVT.2015.2399411

[11] Bellucci, P., Cipriani, E. "Data accuracy on automatic traffic counting: the SMART project results", European Transport Research Review, 2(4), pp. 175-187, 2010. https://doi.org/10.1007/s12544-010-0039-9

[12] Farkas, C., Dán, A. "Stochastic Modeling of Electric Car Charging Station for a Taxi Fleet", Periodica Polytechnica Electrical Engineering and Computer Science, 58(4), pp. 175-181, 2014. https://doi.org/10.3311/PPee.7761

[13] Bodrog, L., Heindl, A., Horváth, G., Telek, M. "A Markovian canonical form of second-order matrix-exponential processes", European Journal of Operational Research, 190(2), pp. 459-477, 2008. https://doi.org/10.1016/j.ejor.2007.06.020

[14] "MATLAB Central File Exchange", [online]. Available at: http:// www.mathworks.com/matlabcentral/fileexchange/34943-fit-all-valid-parametric-probability-distributions-to-data [Accessed: 16 April 2016].

[15] Box, G. E. P., Jenkins, G. M., Reinsel, G. C. "Time Series Analysis: Forecasting and Control", 3rd ed., Prentice Hall, New Jersey, USA, 1994.

[16] Latouche, G., Ramaswami, V. "Introduction to Matrix Analytic Methods in Stochastic Modeling", ASA-SIAM Series on Statistics and Applied Mathematics, Society for Industrial and Applied Mathematics, Philadelphia, USA, 1999. https://doi.org/10.1137/1.9780898719734 